\title{
EFICIÊNCIA PRODUTIVA DE OVELHAS COM DIFERENTES CARACTERÍSTICAS CONFORMACIONAIS SOB PASTEJO
}

\section{PRODUCTIVE EFFICIENCY OF SHEEP WITH DIFFERENT CONFORMATION TRAITS UNDER GRAZING}

\author{
Verônica Gindri Manzoni ${ }^{1}$ \\ Ricardo Zambarda Vaz ${ }^{1^{*}}$ \\ Otoniel Geter Lauz Ferreira ${ }^{1}$ \\ Olmar Antônio Denardin Costa ${ }^{1}$ \\ Fernando Amarilho Silveira ${ }^{1}$ \\ 1Universidade Federal de Pelotas, Pelotas, RS, Brasil \\ *Autor para correspondência - rzvaz@terra.com.br
}

Resumo: Objetivou-se avaliar a eficiência produtiva de ovelhas Corriedale de diferentes condições e estruturas corporais ao parto, mantidas em pastagem natural e/ou cultivada hibernal. Ao parto, as fêmeas foram classificadas em médias (frame $=50$ a $57,5 \mathrm{~kg}$ ) e grandes (frame $=57,6$ a $62 \mathrm{~kg}$ ) quanto ao tamanho e em média ( 2 a 3 pontos) ou alta (3,5 a 4,5 pontos) quanto ao escore de condição corporal. $\mathrm{O}$ delineamento experimental utilizado foi o inteiramente casualizado em arranjo fatorial 2 × 2 (dois grupos de estruturas corporais x duas classes de escore de condição corporal). Ovelhas com frame médio foram mais leves em relação às grandes ao parto $(39,97$ vs 48,16 kg, respectivamente) e ao desmame (43,10 vs $50,22 \mathrm{~kg}$, respectivamente). As eficiências produtivas ao parto e ao desmame não diferiram entre ovelhas médias $(46,91$ e 44,24 kg) e grandes $(47,83$ e 45,69 $\mathrm{kg}$ ), respectivamente. Ovelhas com condição corporal média ao parto quando comparadas com ovelhas de condição corporal alta não apresentaram diferenças significativas para eficiência produtiva ao parto (46,89 vs. $47,82 \mathrm{~kg}$ ) e ao desmame (44,10 vs. $45,83 \mathrm{~kg})$. O tamanho animal e o escore de condição corporal ao parto não influenciam a eficiência produtiva dos rebanhos ovinos sob pastejo.

Palavras-chave: Condição corporal; Corriedale; desmame; frame; parto.

\begin{abstract}
This study was carried out to evaluate the productive efficiency of Corriedale sheep of different conformation traits, reared on natural and/or cultivated winter pastures. At calving, the females were classified as medium (frame $=50$ to $57.5 \mathrm{~kg}$ ) and large (frame $=57.6$ to $62 \mathrm{~kg}$ ) regarding the size and as average ( 2 to 3 points) or high (3.5 to 4.5 points) regarding the body condition score. The experimental design was entirely randomized in a 2 x 2 factorial arrangement (two groups of body weights $x$ two classes of body condition at calving). Sheep with medium body structure were lighter compared to large ones at calving (39.97 vs $48.16 \mathrm{~kg}$, respectively) and at weaning (43.10 vs $50.22 \mathrm{~kg}$, respectively). The production efficiency at calving and at weaning did
\end{abstract}


not differ between medium (46.91 and $44.24 \mathrm{~kg}$ ) and large sheep (47.83 and $45.69 \mathrm{~kg}$ ), respectively. Sheep with average body condition at calving compared with sheep with high body condition showed no significant differences for productive efficiency at calving (46.89 vs. 47.82) and at weaning ( 44.10 vs. 45.83$)$. The animal size and the body condition score at calving did not influence the production efficiency of sheep herds on grazing.

Keywords: Body condition; calving; corriedale; frame; weaning.

Recebido em: 04 abril de 2016

Aceito em: 01 novembro de 2016

\section{Introdução}

A ovinocultura brasileira vem ganhando destaque com a ascensão da produção de carne, encaminhando-se à procura de animais mais eficientes e adaptados, visando maior rentabilidade dos sistemas produtivos. A base alimentar da ovinocultura no Sul do País ainda são as pastagens naturais que, devido às condições climáticas e à diversidade de espécies, oscilam quantitativa e qualitativamente no decorrer dos ciclos produtivos. Além disso, o mau manejo empregado nas mesmas ocasiona baixas lotações resultando em queda na produtividade ${ }^{(1)}$, limitando a produção animal em períodos como final da gestação e início da lactação ${ }^{(2)}$.

O índice mais utilizado para avaliar rebanhos ovinos de cria é a taxa de desmame; entretanto, somente o percentual de cordeiros desmamados em relação ao número de ovelhas não tem consistência, pois a quantidade deve estar associada à qualidade da produção. Desta forma, o peso dos cordeiros ao desmame e os quilogramas de cordeiros por ovelhas mantidas no rebanho são indicadores mais eficientes para a avaliação e tomada de decisão nos sistemas produtivos.

O escore de condição corporal é um dos parâmetros indiretos mais utilizados para se predizer a quantidade de reservas corporais em ruminantes, uma vez que possui uma correlação positiva e significativa $(\mathrm{r}=0,20 ; \mathrm{P}<0,05)$ com o depósito de gordura subcutânea ${ }^{(3)}$. Estas reservas ao parto são importantes, pois indicam o que o animal dispõe no momento para manter a lactação em níveis satisfatórios de produção, refletindo no desenvolvimento e peso dos cordeiros ao nascer e ao desmame $^{(4)}$. Os baixos escores podem afetar o desenvolvimento dos animais, bem como o desempenho reprodutivo subsequente.

São escassos os estudos que relatam a influência da estrutura corporal nos parâmetros de eficiência produtiva do rebanho ovino ${ }^{(5)}$. A partir daí surge a necessidade de investigações que demonstrem essa relação, visando adequar os animais ao ambiente, tentando maximizar a interação genótipo $\mathrm{x}$ ambiente.

O objetivo do presente estudo foi avaliar a eficiência produtiva do parto à desmama de ovelhas cruzas Corriedale sob pastejo com diferentes estruturas e condições corporais ao parto. 


\section{Material e Métodos}

O experimento foi desenvolvido no Centro Agropecuário da Palma - Universidade Federal de Pelotas, localizado na região Sul do Estado do Rio Grande do Sul. O clima da região e Cfa (subtropical úmido).

Foram utilizadas 58 ovelhas prenhes de diferentes idades, cruzas Corriedale (Código CEEA/UFPEL: 8878/2014). As parições ocorreram de 20 de agosto a 10 de setembro, sendo todas as gestações simples. Ao parto, as ovelhas foram classificadas, segundo suas características conformacionais, em médias (frame $=50$ a $57,5 \mathrm{~kg}$ ) e grandes (frame $=57,6$ a $62 \mathrm{~kg}$ ) ${ }^{(5)} \mathrm{e}$, de acordo com a condição corporal ao parto, em média (2,5 a 3,0 pontos) e alta $(\geq 3,5 \text { a 4,5 pontos })^{(6)}$.

A estrutura corporal foi calculada por meio das medidas de peso corporal (PC), comprimento corporal (CC), altura do anterior (ALTA) e altura do posterior (ALTP), segundo a fórmula: $\mathrm{FRAME}=[(\mathrm{PC}+\mathrm{CC}+\mathrm{ALTA}+\mathrm{ALTP}) / 4]^{(5)}$. Os pesos corporais foram obtidos com balança mecânica apropriada para pequenos animais, com capacidade de $500 \mathrm{~kg}$ e divisão mínima de $100 \mathrm{~g}$, sendo determinados ao parto, ao desmame e periodicamente a cada 21 dias. As mensurações de CC, ALTA e ALTP foram realizadas estando os animais estáticos em uma estação (superfície horizontal) e com o uso de fita métrica graduada em centímetros compreendendo as seguintes distâncias: CC - entre a cernelha e a garupa; ALTA - entre a cernelha e o solo; e ALTP - entre a cabeça do fêmur e o solo ${ }^{(7)}$.

A condição corporal ao parto foi determinada pela palpação da região dorso lombar da coluna vertebral, verificando a deposição de gordura e músculo encontrada no ângulo formado pelos processos dorsais e transversos, utilizando a escala de 1 (muito pobre) a 5 (excelente) ${ }^{(6)}$.

O manejo sanitário para controle da verminose anterior ao período experimental foi realizado de forma estratégica com produtos de longo espectro, a fim de que não fosse prejudicial ao desempenho dos animais e de evitar manejo das ovelhas durante a gestação. Ao início do experimento e durante o mesmo, a infestação endoparasitária foi monitorada com intervalos médios de 14 dias, por meio de exame de contagem do número de ovos por grama de fezes (OPG) pela técnica de McMaster $^{(8)}$, sendo realizadas dosificações sempre que a média de OPG dos animais superasse 500 ovos por grama de fezes. O calendário de vacinações adotado esteve de acordo com as determinações da Secretaria da Agricultura do Estado do Rio Grande do Sul. A vacinação contra as clostridioses foram realizadas anteriormente ao parto nas ovelhas e aos quarenta e cinco dias após o nascimento nos cordeiros.

Após o nascimento, as mães e crias foram pesadas, os cordeiros foram identificados com brincos plásticos na orelha esquerda e permaneceram juntamente com as suas mães até a data do desmame, realizado em média aos 80 dias de idade. Depois do nascimento tomou-se cuidado para que os cordeiros ingerissem o colostro. Em seguida, foi realizado o corte do umbigo, com tesoura desinfetada, e imersão em tintura de iodo a 5\%, segurando-se o cordão umbilical em um frasco de boca larga por 40 a 60 segundos. 
Os animais foram manejados em lote único em regime de pastejo num sistema de rodízio de potreiros compostos de pastagens naturais ou cultivada de inverno, de acordo com as disponibilidades. Manteve-se um nível de oferta não limitante ao consumo dos animais e a perpetuação das pastagens. Devido ao alto grau de infestação de endoparasitas, buscou-se evitar ao máximo a influência destes no desempenho animal.

A composição botânica das pastagens naturais é diversificada, estando gramíneas e compostas misturadas. As espécies predominantes de gramíneas são Paspalum notatum nas partes mais altas, Axonopus fissifolius em partes mais úmidas, destacando-se a Andropogon lateralis extrato como superior. Entre as leguminosas verificou-se a presença de alguns trevos, mas com grande ênfase a família Desmodium. A pastagem de inverno era composta apenas de azevém comum (Lolium multiflorum Lam.).

Em todos os períodos foram realizadas avaliações de oferta de forragem em matéria seca (MS), de proteína bruta $(\mathrm{PB})$ e fibra em detergente neutro (FDN). As estimativas da oferta de forragem disponível foram realizadas pelo método comparativo ${ }^{(9)}$ a cada 21 dias, com as amostras retiradas para a determinação da disponibilidade da pastagem. As amostras obtidas foram armazenadas para posteriores análises laboratoriais. Os potreiros de pastagem natural foram manejados na média com $890 \mathrm{~kg}$ de MS, com a qualidade proteica variando nos períodos entre 8,04 e 10,70\% de PB e entre 53,76 e $61,41 \%$ de FDN. Já a pastagem de azevém apresentou oferta média de $1320 \mathrm{~kg}$ de MS/ha durante o período experimental, com variações entre 1090 e $1640 \mathrm{~kg}$ de MS durante os períodos. O teor de PB e FDN da pastagem de azevém variaram de 11,4 e 17,8\% e de 47,12 e 57,89\%, respectivamente, durante os períodos avaliados.

Para a eficiência produtiva foram avaliados os pesos ao parto e ao desmame (média 80 dias) das ovelhas e cordeiros. As variações de pesos corporais compreendidos entre o parto e o desmame foram determinados por meio da diferença entre as pesagens divididas pelo período de avaliação.

Foram avaliadas as eficiências produtivas das ovelhas ao parto (EPP) e ao desmame (EPD), de acordo com metodologia adaptada para ovinos ${ }^{(10)}$. As estimativas foram consideradas em relação à quantidade de $\mathrm{kg}$ de cordeiros desmamados para cada $100 \mathrm{~kg}$ de ovelhas paridas e desmamadas. A EPP foi calculada por meio da relação entre peso ao desmame do cordeiro e peso ao parto da ovelha, já a EPD foi calculada por meio da relação entre peso ao desmame do cordeiro e peso ao desmame da ovelha.

O delineamento experimental foi o inteiramente casualizado em arranjo fatorial 2 × 2 (dois grupos de características conformacionais x duas classes de escore de condição corporal, ambos ao parto) e os resultados submetidos à análise de variância e teste F de acordo com o seguinte modelo:

$$
\mathrm{Y}_{\mathrm{ijklm}}=\mu+\mathrm{FRAME}_{\mathrm{i}}+\mathrm{ECC}_{\mathrm{j}}+\left(\mathrm{FRAME}^{*} \mathrm{CC}\right)_{\mathrm{ij}}+\mathrm{IC}_{\mathrm{k}}+\mathrm{IO}_{\mathrm{l}}+\mathrm{S}_{\mathrm{m}}+\mathrm{E}_{\mathrm{ijklm}}
$$

Em que: $\mathrm{Y}_{\mathrm{ijklm}}=$ variáveis dependentes; $\mu=$ médias observadas; FRAME $\mathrm{F}_{\mathrm{i}}=$ efeito da estrutura corporal de ordem $\mathrm{i}$, sendo $\mathrm{i}=1$ (médio); 2 (grandes); $\mathrm{CC}_{\mathrm{j}}=$ efeito de escore de condição corporal de ordem $\mathrm{j}$, sendo $\mathrm{j}=1$ (média) e 2 (alta); $\left(\mathrm{FRAME}^{*} \mathrm{CC}\right)_{\mathrm{ij}}=$ interação entre a estrutura corporal de 
ordem i e o escore de condição corporal de ordem $\mathrm{j} ; \mathrm{IN}_{\mathrm{k}}=$ Covariável idade do cordeiro de ordem $\mathrm{k}$; $\mathrm{IO}_{1}=$ Covariável idade da ovelha ao parto ordem $1 ; \mathrm{S}_{\mathrm{m}}=$ covariável sexo dos cordeiros de ordem m; $\mathrm{E}_{\mathrm{ijklm}}=$ Erro aleatório assumindo distribuição normal com média igual a zero e variância $\sigma^{2}$. Como a interação $\mathrm{FRAME*CC}$ foi de baixa magnitude, a mesma foi removida do modelo estatístico final. Quando o $\mathrm{F}$ foi significativo $(\mathrm{P} \leq 0,05)$, foi utilizado o teste de comparação de médias com base no teste " $t$ " no mesmo nível de significância do teste $\mathrm{F}$.

\section{Resultados e discussão}

Por não ter ocorrido interação significativa, os resultados de tamanho de estrutura corporal e condição corporal ao parto foram apresentados e discutidos em separado. A classificação dos grupos de acordo com a estrutura corporal das ovelhas determinou diferenças de pesos corporais das ovelhas ao parto, com superioridade das ovelhas grandes quando comparadas às médias. Estas diferenças mantiveram-se até o desmame dos cordeiros, com superioridade de 17,0\% ao parto e $16,5 \%$ ao desmame, e tais superioridades não influenciaram o peso dos cordeiros ao nascimento e ao desmame (Tabela 1).

Tabela 1. Médias e erros-padrão para caracteristicas de desenvolvimento, desempenho e eficiência produtiva de ovelhas cruzas Corriedale e seus cordeiros com diferentes tamanhos de estrutura corporal

\begin{tabular}{lccc}
\hline & \multicolumn{2}{c}{ Tamanho de estrutura corporal } & \\
\cline { 2 - 3 } & Médias $(\mathbf{n}=\mathbf{2 8})$ & Grandes $(\mathbf{n}=\mathbf{3 0})$ & Pr>F \\
\hline Pesos, $k g$ & $39,97 \pm 1,42$ & $48,16 \pm 1,39$ & 0,0006 \\
Ovelha ao parto & $43,10 \pm 1,04$ & $50,22 \pm 1,08$ & 0,0002 \\
Ovelha ao desmame & $4,46 \pm 0,23$ & $4,69 \pm 0,23$ & 0,4970 \\
Cordeiro nascimento & $18,54 \pm 1,46$ & $22,72 \pm 1,48$ & 0,0657 \\
Cordeiro ao desmame & & & \\
\hline Ganho médio diário, $k g /$ dia & $0,03 \pm 0,02$ & $0,03 \pm 0,02$ & 0,9114 \\
Ovelha na lactação & $0,20 \pm 0,02$ & $0,25 \pm 0,02$ & 0,0864 \\
Cordeiro na lactação & $-0,00 \pm 0,00$ & $-0,01 \pm 0,00$ & 0,3506 \\
Cordeiro pós-desmame & $46,91 \pm 3,86$ & $47,83 \pm 3,93$ & 0,8775 \\
\hline Eficiéncias produtivas, $k g$ de cordeiro/ $100 \mathrm{~kg}$ de ovelhas & & 0,7851 \\
Ao parto & $44,24 \pm 3,45$ & $45,69 \pm 3,59$ & \\
Ao desmame & & & 0,7420 \\
\hline Escore de condição corporal & $2,78 \pm 0,09$ & $2,82 \pm 0,09$ & 0,9189 \\
Ao parto & $2,66 \pm 0,17$ & $2,69 \pm 0,17$ & \\
Ao desmame & & & \\
\hline
\end{tabular}

O tamanho corporal determinado por meio de medidas é mais preciso do que avaliações de escores subjetivos, podendo determinar e associar em termos quantitativos se os animais ganham ou perdem condição corporal em determinados períodos durante as fases de criação. A importância do tamanho 
corporal na produção dos animais reside em sua maior ou menor exigência nutricional ${ }^{(11)}$. Em situações nutricionais não ideais, este fato potencializa baixas produtividades, devido aos animais mais pesados serem mais exigentes e consumirem mais alimentos. No início da gestação, ovelhas consideradas grandes $(>50 \mathrm{~kg}$ ) apresentaram exigência nutricional em proteína e energia maior $\left(15,6\right.$ e $13,3 \%$, respectivamente) ${ }^{(12)}$ do que ovelhas médias (>40 kg). Além disso, animais de maior estrutura também apresentam maiores exigências de consumo de matéria seca, que é determinado em percentual do peso corporal, diminuindo a produtividade dos rebanhos em condições de escassez e ou limitação de alimentos. Nestas circunstâncias, animais menores são mais eficientes ${ }^{(5)}$.

O período de lactação é a fase de maior exigência nutricional da ovelha e, normalmente, as fêmeas sofrem declínio no peso e estado corporal durante este período, devido ao estado físiológico, chamado de balanço energético negativo. Este estado é decorrente da degradação de reservas corporais, quando os nutrientes da dieta alimentar não suprem as exigências do animal, sendo os nutrientes levados ao sangue e posteriormente convertidos na produção de leite para alimentação do cordeiro.

Em contraponto, não houve perda de peso das ovelhas, ao contrário do que normalmente acontece nesta fase, ocorrendo ganho de peso, embora de baixa magnitude (35 e 39 gramas/dia, respectivamente, para médias e grandes). Em avaliação do desempenho de ovelhas Suffolk em pastagens cultivadas em diferentes sistemas de criação ${ }^{(17)}$, ovelhas mantidas em pasto com cordeiros recebendo ou não suplementação perderam peso (-0,05 e -0,31 g/dia, respectivamente) durante o período experimental. Esses resultados foram devido ao fato de as pastagens não suprirem as exigências de mantença e produção de leite.

Ovelhas com maior peso corporal ao parto são mais propensas a parirem cordeiros mais pesados, referindo-se, neste caso, à estrutura corporal grande. No entanto, neste estudo não foram encontradas diferenças estatísticas, não comprovando este fato. Fêmeas mais pesadas e, consequentemente, com melhor condição corporal, produzem mais leite em relação a animais de peso e estado corporal inferior ${ }^{(21)}$, o que não demonstrou influenciar os resultados obtidos, não ocorrendo diferenças entre as classes para pesos ao nascimento e ao desmame dos cordeiros.

Ao nascimento, os cordeiros não apresentaram diferenças no peso entre as estruturas corporais; entretanto, cordeiros filhos de ovelhas de maior peso apresentaram em média 0,23 kg a mais em relação aos filhos de ovelhas médias. O peso ao nascimento dos cordeiros foram superiores aos observados em experimento semelhante ${ }^{(13)}$, que revelou média de 3,54 x 3,12 $\mathrm{kg}$ em cordeiros Corriedale e Ideal, respectivamente, quando mantidos em pastagem natural melhorada, sendo estes pesos considerados como bons e determinantes de altos índices de sobrevivência.

Os pesos ao nascimento de pequenos ruminantes podem ser influenciados pelo grupo genético, pelo número de cordeiros, pela nutrição da fêmea e pelo sexo ${ }^{(11,14,15)}$. Destes fatores, a alimentação é limitante, mas pode ser manipulada e o manejo das forrageiras é decisivo para os avanços corporais $^{(16)}$.

Apesar de não ocorrerem diferenças significativas ao desmame (80 dias), os cordeiros filhos das ovelhas de estrutura corporal grande apresentaram incremento de 22,54\% no peso ao desmame em relação aos cordeiros das ovelhas de estrutura corporal média. Em desmama de cordeiros cruzados, aos 45 ou 60 dias de idade ${ }^{(17)}$, foram verificados pesos inferiores para os animais desmamados aos 
45 dias em relação aos desmamados aos 60 dias, com valores de 19,00 e 23,30 kg, respectivamente. Este peso menor que o encontrado no presente estudo se deve à menor idade de desmame dos cordeiros e ao sistema alimentar inadequado que corrobora para o menor desempenho.

Estes fatos evidenciam que a extensão do período de lactação determina maiores pesos dos cordeiros ao desmame. Contudo, em condições adversas, este fato é prejudicial à ovelha, ocasiona maior desgaste e pode até comprometer seu desempenho reprodutivo futuro, agravando-se à medida em que o nível nutricional torna-se não ideal ${ }^{(18)}$.

O campo nativo sob condições adequadas de manejo pode proporcionar desempenhos satisfatórios à desmama de cordeiros, sem afetar a estrutura corporal das mães ${ }^{(19)}$. Apesar de as ovelhas grandes serem mais pesadas ao parto e ao desmame, as mesmas não foram mais eficientes nas avaliações produtivas quando comparadas com as de estrutura médias. Normalmente, em outras espécies de ruminantes, como as vacas de corte, as fêmeas de tamanho menor são mais eficientes na produção de $\mathrm{kg}$ de bezerros, em relação às fêmeas grandes, principalmente em condições de escassez alimentar $^{(20)}$.

A não diferença nas eficiências produtivas ao parto e ao desmame entre os tamanhos dos animais demonstra existirem animais mais adaptados para determinados ambientes. Esses resultados refletem na possibilidade de escolha e seleção de animais mais produtivos para os diferentes sistemas de produção, em busca de produtividades maiores. Em outras palavras, animais de menor porte consomem menos alimentos, podendo assim se colocar mais animais na mesma área obtendose maior produção. Na média, a alimentação disponibilizada às ovelhas parece ter sido suficiente para atender à demanda alimentar de manutenção e produção de leite, ao passo que ao final do período de aleitamento, as ovelhas apresentaram pequeno aumento de ganho de peso, com aumentos de 2,67 e 2,14 kg para estrutura corporal, médias e grandes, respectivamente.

Os pesos ao parto e ao desmame das ovelhas e cordeiros não diferiram nos grupos com diferentes classes de escores condições corporais ao parto (Tabela 2). Entretanto, esperava-se que as ovelhas com maior deposição de músculo e gordura corporal também apresentassem maiores pesos corporais, desde que mantidas as mesmas estruturas corporais. Variações por diferenças de classes ou ainda pela subjetividade do método podem ocorrer $^{(7)}$, pois baixas condições influenciam de forma mais acentuada os pesos das ovelhas ao parto e nascimento dos cordeiros ${ }^{(17)}$. Porém este fato não ocorreu no presente estudo.

Os pesos ao nascimento e ao desmame dos cordeiros não diferiram entre as classes de condições corporais (Tabela 2). Era esperado que ovelhas com mais reservas parissem e desmamassem cordeiros mais pesados. O peso do cordeiro ao nascimento e ao desmame está linearmente relacionado à condição corporal da mãe, de maneira que o animal com melhor condição corporal é capaz de produzir mais leite e cordeiros mais pesados ${ }^{(4)}$.

O escore de condição corporal da ovelha é influenciado pelo nível nutricional ao qual a mesma está exposta, sendo fundamental para o crescimento do cordeiro na fase de aleitamento. Neste período o animal jovem tem a maior velocidade de crescimento em ganho de peso, pois o alimento principal é o leite, que constitui todos os nutrientes necessários para o devido desempenho corporal ${ }^{(21)}$. Em estudo com ovelhas cruzas Ile de France mantidas em pastagem cultivada e recebendo suplemento antes e após o parto a nível de $1 \%$ do peso corporal $^{(4)}$, foi verificado que as fêmeas de melhores 
escores de condição corporal ao parto pariram cordeiros mais pesados, mantiveram o peso durante a lactação e desmamaram cordeiros maiores.

Tabela 2. Médias e erros-padrão para características de desenvolvimento, desempenho e eficiência produtiva de ovelhas cruzas Corriedale e seus cordeiros com diferentes condições corporais ao parto

\begin{tabular}{|c|c|c|c|}
\hline & Condição corp & ral ao parto & \\
\hline & Média $(n=26)$ & Alta $(n=32)$ & $P r>F$ \\
\hline Pesos, $\mathrm{kg}$ & & & \\
\hline Ovelha ao parto & $42,50=1,29$ & $45,64=1,34$ & 0,1515 \\
\hline Ovelha ao desmame & $45,06 \pm 1,15$ & $48,26=0,96$ & 0,0573 \\
\hline Cordeiro nascimento & $4,74=0,24$ & $4,40=0,22$ & 0,3325 \\
\hline Cordeiro ao desmame & $19,70=1,61$ & $21,56=1,37$ & 0,4119 \\
\hline Ganho médio diário, $k g$ & & & \\
\hline Ovelha na lactação & $0,04=0,02$ & $0,03=0,02$ & 0,7577 \\
\hline Cordeiro na lactação & $0,21=0,02$ & $0,24=0,01$ & 0,2938 \\
\hline Cordeiro pós desmame & $-0,00 \pm 0,00$ & $-0,01 \pm 0,00$ & 0,7353 \\
\hline Eficiências produtivas, & $\mathrm{kg}$ de ovelha & & \\
\hline Ao parto & $46,89 \pm 4,26$ & $47,82=3,64$ & 0,8757 \\
\hline Ao desmame & $44,10 \pm 3,81$ & $45,83=3,21$ & 0,7463 \\
\hline Escore de condição Cor & & & \\
\hline Ao parto & $2,56 \pm 0,10$ & $3,50=0,09$ & 0,0017 \\
\hline Ao desmame & $2,51=0,16$ & $2,85=0,15$ & 0,1475 \\
\hline
\end{tabular}

As variações de pesos corporais tanto para ovelhas em condições médias e altas $(0,042$ vs $0,031 \mathrm{~kg}$, respectivamente) e seus cordeiros (0,213 vs. 0,246 kg, respectivamente) não diferiram durante o período de lactação. Este resultado assemelha-se a outro encontrado na literatura ${ }^{(17)}$, afirmando que a condição corporal ao parto da ovelha influência na taxa de sobrevivência dos cordeiros.

A eficiência produtiva indica a quantidade de quilogramas produzidos de cordeiros para cada 100 $\mathrm{kg}$ de ovelha. A eficiência produtiva pode ser calculada ao parto, bem como ao desmame, em função do peso do cordeiro ao desmame relacionado ao peso das ovelhas no parto e no desmame. As ovelhas de diferentes classes de escores de condição corporal ao parto não diferiram nas suas eficiências ao parto e ao desmame com valores de 46,89 e 44,10 kg e 47,82 e 45,83 kg para ovelhas de classificação médias e altas, respectivamente. Este fato demonstra que a condição corporal ao parto não é determinante na produtividade dos rebanhos quando esta está classificada como média ou mais, gerando quilos do produto cordeiro de forma semelhante. Esta diferença pode ser determinante na rentabilidade dos sistemas produtivos, pois pode representar menor período de terminação dos animais, bem como giro e retorno do capital investido mais rápido ${ }^{(22)}$, aumentando as receitas e diluindo os custos fixos fatores determinantes de melhores rentabilidades. Estes fatores se acentuam à medida que os sistemas produtivos tornam-se mais intensivos, tendo a necessidade de aumento de produtividade e eficiência ${ }^{(23)}$. 
No entanto, para manter os mesmos níveis de eficiência, as ovelhas com melhor escore de condição corporal ao parto tiveram maiores perdas de condicionamento corporal, o que promoveu similaridade para esta variável ao desmame. Tal fato retrata que as ovelhas maiores e de melhores condições corporais tiveram que recorrer às suas reservas energéticas para manter o mesmo nível de produção. É interessante ressaltar que, embora não significativas as diferenças entre o peso ao nascimento e ao desmame dos cordeiros (Tabela 2), os filhos das ovelhas de condição corporal alta tiveram um ganho de peso no período de lactação de 17,16 kg vs 14,96 kg das médias. Desta forma, além de as ovelhas maiores exigirem mais nutrientes do que as demais, elas provavelmente produzem mais leite, o que pode ter sido a provável razão da maior perda de escore dessa categoria. Ovelhas que parem em melhor condição corporal produzem mais leite, com maiores pesos dos cordeiros ao desmame ${ }^{(14)}$. No entanto, se a alimentação for restrita e o animal tiver de recorrer às suas reservas energéticas, podem ocorrer prejuízos na reprodução subsequente em sistemas mais intensivos de produção ${ }^{(21)}$.

Nos primeiros 30 dias após o desmame dos animais, ocorreu perda de peso dos cordeiros, independente da classificação ao parto em tamanho ou escore de condição corporal das ovelhas, tendo os mesmos variações negativas em média de $0,010 \mathrm{~kg} \mathrm{dia}^{-1}$. Os estresses desses animais na troca de alimentação com a retirada do leite, mais a ausência da mãe, contribuíram para o declínio do peso até sua adaptação completa ao novo manejo alimentar, na transição de pseudo-ruminante para ruminante, sendo os animais influenciados de forma mais acentuada à medida que se reduz a idade de desmame, em função da maior dependência dos cordeiros do leite materno para a sua alimentação. Existem algumas maneiras de se amenizar este distúrbio, como a adoção de estratégias de adaptação à suplementação antes do período de desmame como o creep-feeding que é uma alternativa de tecnologia fundamental para a manutenção e/ou ganho de peso dos cordeiros, diminuindo, assim, seu estresse e seu tempo de permanência na propriedade ${ }^{(21)}$.

\section{Conclusão}

Ovelhas cruza Corriedale, de características conformacionais grandes, são mais pesadas ao parto e ao desmame quando comparadas a ovelhas de estrutura corporal média, não diferindo nas eficiências produtivas de cordeiros. Escores de condição corporal ao parto médio e alto não influenciam o desempenho e as eficiências produtivas de ovelhas e cordeiros cruza Corriedale sob pastejo, embora ovelhas com escore alto percam mais acentuadamente essa condição no período de lactação que aquelas de escore médio.

\section{Referências}

1. Poli CHEC, Monteiro ALG, Barros CS, Moraes A, Fernandes MAM, Piazzetta HVL. Produção de ovinos de corte em quatro sistemas de produção. Revista Brasileira de Zootecnia. 2008, 37 (4): 666-673. 
2. Farinatti LHE, Rocha MG, Poli CHEC, Pires CC, Pötter L, Silva JHS. Desempenho de ovinos recebendo suplementos ou mantidos exclusivamente em pastagem de azevém (Lolium multiflorum Lam.). Revista Brasileira de Zootecnia. 2006, 35(2):527-534.

3. Mendizabal JA, Delfa R, Arana A, Eguinoa P, Purrov A. Lipogenic activity in goats (Blanca Celtibérica) with different body condition scores. Small Ruminant Research. 2007, 67: 285-290.

4. Rosa GT, Siqueira ER, Gallo SB, Moraes SSS. Influência da suplementação no pré-parto e da idade de desmama sobre o desempenho de cordeiros terminados em confinamento. Revista Brasileira de Zootecnia. 2007, 36 (4): 953-959.

5. Souza Junior EL, Sousa WH, Pimenta Filho EC, Gonzaga Neto S, Cartaxo FQ, Cezar MF, Cunha MGG, Pereira Filho JM. Effect of frame size on performance and carcass traits of Santa Inês lambs finished in a feedlot. Revista Brasileira de Zootecnia. 2013, 42 (4): 284-290.

6. Osório JCS, Osório MTM, Jardim POC. Métodos para avaliação de carne ovina in vivo na carcaça e na carne. 1st ed. Pelotas: Editora e Gráfica Universitária - UFPel, 1998. p. 107. Portuguese.

7. Osório JCS, Osório MTM. Produção de carne ovina: técnicas de avaliação in vivo e na carcaça. 1st ed. Pelotas: Editora e Gráfica Universitária - UFPel, 2003. 73p. Portuguese.

8. Gordon HML, Whitlock AV. A new technique for counting nematode eggs in sheep feces. Journal Council Scientific Industrial Research. 1939, 12: 50-52.

9. Haydock KP, Shaw NH. The comparative yield method for stimating dry matter yield of pasture. Australian Journal of Experimental Agriculture and Animal Husbandry. V. 15, n.76, p. 663-670. 1975.

10. Ribeiro ELA, Restle J, Da Rocha MA, Mizubuti I Y, Silva LDDF. Eficiência produtiva em vacas primíparas das raças Aberdeen Angus e Charolês. Revista Brasileira de Zootecnia. 2001, 30 (1): 125-132.

11. Menezes JJL, Gonçalves HC, Ribeiro MS, Rodrigues L, Cañizares GIL, Medeiros BBL, Giassetti AP. Desempenho e medidas biométricas de caprinos de diferentes grupos raciais. Revista Brasileira de Zootecnia. 2007, 36 (3): 635-642.

12. NRC - NATIONAL RESEARCH COUNCIL. Nutrient requirements of sheep. 6.ed. Washington, D.C.: National Academy Press, 1985. 99p

13. Rech CLS, Rech JL, Fischer V, Osório MTM, Manzoni N, Moreira H, LM, Silveira IDB, Tarouco AK. Temperamento e comportamento materno-filial de ovinos das raças Corriedale e Ideal e sua relação com a sobrevivência dos cordeiros. Ciência Rural. 2008, 38 (5): 1388-1393.

14. Castro FAB, Ribeiro ELA, Koritiaki NA, Mizubuti IY, Silva LDF, Pereira ES, Pinto AP, Constantino C, Fernandes Junior F. Desempenho de cordeiros Santa Inês do nascimento ao desmame filhos de ovelhas alimentadas com diferentes níveis de energia. Semina: Ciências Agrárias. 2012, 33(6 supl 2): 3379-3388.

15. Mexia AA, Macedo FAF, Macedo RMG, Sakaguti ES, Santello GA, Capovilla LCT, Zundt M, Sasa A. Desempenho e características das fibras musculares esqueléticas de cordeiros nascidos de ovelhas que receberam suplementação alimentar em diferentes períodos da gestação. Revista Brasileira de Zootecnia. 2006, 35 (4): 1780-1787.

16. Castro FAB, Ribeiro ELA, Mizubuti IY, Silva LDF, Barbosa MAAF, Marson B, Grandis FA, Fernandes Junior F, Pereira ES. Energia dietética ao final da gestação e durante a lactação e desempenho de ovinos Santa Inês em sistema de acasalamento acelerado. Semina: Ciências Agrárias. 2013, 34(6 supl 2): $4187-$ 4202. 
17. Ribeiro LAO, Brito MA, Mattos RC. Ewes shorn and unshorn during pregnancy in South Brazil: effects on body condition score and lamb birth weight. Brazilian Journal of Veterinary Research and Animal Science.2010, 42 (2): 111-117.

18. Nasciutti NR, Tsuruta SA, Oliveira RSBR, Bisinoto M, Headley SA, Mundim AV, Noleto PG, Saut JPE. Perfil metabólico em ovelhas Santa Inês, com baixo escore de condição corporal, no periparto. Boletim da Indústria Animal. 2012, 69 (2): 137-145.

19. Motta JF, Ferreira OGL, Vaz RZ, Costa OAD, Alonzo LAG, Gonçalves MS, Lopes AG, Pedra WU. Desempenho produtivo e qualidade da carcaça de cordeiros avaliados em dois sistemas alimentares. Boletim de Indústria Animal. 2016, 73(1): 15-23.

20. Vaz RZ, Restle J, Pacheco PS, Vaz FN, Alves Filho DC, Brondani IL, Pascoal LL, Argenta FM. Produtividade e eficiência de produção de vacas de diferentes grupos genéticos submetidas a pastagens cultivadas no pré ou pós-parto. Semina: Ciências Agrárias. 2014, 35 (5): 2697-2708.

21. Boucinhas CC, Siqueira ER, Maestá SA. Dinâmica do peso e da condição corporal e eficiência reprodutiva de ovelhas da raça Santa Inês e mestiças Santa Inês-Suffolk submetidas a dois sistemas de alimentação em intervalos entre partos de 8 meses. Ciência Rural. 2006, 36(3): 904-909.

22. Barros CS, Monteiro ALG, Poli CHEC, Dittrich JR, Canziani JRF, Fernandes MAM. Rentabilidade da produção de ovinos de corte em pastagem e em confinamento. Revista Brasileira de Zootecnia. 2009, 38(11): 2270-2279.

23. Aita MF, Fischer V, Poli CHEC, Osório MTM, Silveira IDB, Selbot MB, Montano DB, Losekann PB. Relação entre o escore de comportamento materno e as características fisiológicas de ovelhas. Revista Brasileira de Zootecnia. 2012, 41(4):1035-1043. 